\title{
EFEITO DE DIFERENTES VOLUMES DE CALDA NO RALEIO QUÍMICO DE FRUTOS DA MACIEIRA 'GALA'1
}

\author{
ANÍSIO PEDRO CAMILO² e LUIZ ANTONIO PALLADIN ${ }^{3}$
}

\begin{abstract}
RESUMO - O objetivo deste trabalho foi comparar a eficiência de diferentes volumes de calda de raleantes químicos em plantas de macieira (Malus domestica Borkh), cultivar Gala. Foram utilizados o ácido naftalenoacético (ANA) (15 g/ha de i.a.) e o carbaryl (1-naftil-N-metilcarbamato) (1.000 g/ha de i.a.) aplicando-se 16, 300, 430, 950, 1.300 e $1.900 \mathrm{~L} / \mathrm{ha}$ de calda. O ANA foi aplicado cinco dias após a plena floração (DAPF), e o carbaryl, aos 15 DAPF. O volume de calda não parece ter sido o fator mais importante para a eficiência do raleio: os tratamentos utilizados provocaram respostas semelhantes nas plantas. O peso e a porcentagem de frutas $\geq 66 \mathrm{~mm}$, em todos os tratamentos, com exceção de $430 \mathrm{~L} / \mathrm{ha}$, foram maiores do que os da testemunha, mas não diferiram entre si.
\end{abstract}

Termos para indexação: Malus domestica, maçã, ácido naftalenoacético, carbaryl, substâncias de crescimento vegetal.

\section{EFFECT OF FRUIT THINNERS AT DIFFERENT VOLUMES ON 'GALA' APPLE TREE}

\begin{abstract}
The aim of this trial was to compare the thinning efficiency of chemicals sprayed at different volumes, in apple (Malus domestica Borkh), cultivar Gala. Naphthaleneacetic acid (NAA) (15 g/ha of a.i.) and carbaryl (1-naphthyl-N-methylcarbamate) $(1,000 \mathrm{~g} / \mathrm{ha}$ of a.i.) were used in spray solutions at $16,300,430,950,1,300$ and 1,900 L/ha maintaining the dose of each product per hectare. NAA was sprayed at five days after full bloom (DAFB) and carbaryl at 15 DAFB. The volume did not play a major role on the efficiency of the treatments on fruit thinning: the different spray volumes showed similar thinning efficiency on 'Gala' apple. Except at $430 \mathrm{~L} / \mathrm{ha}$, chemical thinners sprayed at different volumes gave fruit weight and percentage of fruits $\geq 66 \mathrm{~mm}$ greater than the control, but did not differ among them.
\end{abstract}

Index terms: Malus domestica, apples, NAA, carbaryl, plant growth substances.

\section{INTRODUÇÃO}

O raleio químico está amplamente difundido em todas as regiões produtoras de maçãs. Esta técnica é de aplicação mais prática, rápida e econômica, quando comparada com o raleio manual. Como o raleio químico é efetuado no início do desenvolvimento das frutas, reduz a competição entre elas já neste período. Assim, as frutas remanescentes serão de maior tamanho e as chances de alternância na produção são reduzidas (Edgerton, 1973; Ebert et al.,

\footnotetext{
${ }^{1}$ Aceito para publicação em 20 de março de 2000.

${ }^{2}$ Eng.Agrôn., Ph.D., Embrapa/Empresa de Pesquisa Agropecuária e Extensão Rural de Santa Catarina (EPAGRI)Estação Experimental de Caçador, Caixa Postal 591, CEP 89500-000 Caçador, SC.

E-mail: anisiocamilo@conection.com.br

${ }^{3}$ Eng.Agrôn., M.Sc., EPAGRI.

E-mail: palladin@unc-cdr.rct-sc.br
}

1988; Faust, 1989; Espada Carbó, 1994). Além do efeito no tamanho, um bom raleio proporciona frutas mais coloridas, com maior teor de açúcar e mantém o vigor da planta.

Os raleantes químicos mais utilizados no Brasil são: ácido naftalenoacético (ANA) e carbaryl (Camilo et al., 1991, 1992). Os produtos usados como raleantes químicos não são sistêmicos (Giulivo et al., 1981; Nir \& Lavee, 1981). Desta forma, cada cacho floral tem de ser atingido pelo produto para que este seja efetivo. A eficiência do raleio também depende da dosagem adequada e da deposição uniforme do raleante na planta.

A adição de espalhante adesivo ou de óleo mineral permite melhor absorção dos raleantes químicos, podendo-se até reduzir as concentrações desses produtos (Wertheim, 1986).

Como nas aplicações de fungicidas e inseticidas em culturas arbóreas, as dos raleantes químicos têm 
sido realizadas com alto volume de calda, ou seja, molhando-se as plantas até o ponto de gotejamento. Isto causa perda de produtos para o solo e contribui para a contaminação do meio ambiente.

Os volumes de calda para culturas arbóreas são classificados por Matthews (1982) em alto volume (mais de $1.000 \mathrm{~L} / \mathrm{ha}$ ), médio (entre 500 e $1.000 \mathrm{~L} / \mathrm{ha}$ ), baixo (entre 200 e $500 \mathrm{~L} / \mathrm{ha}$ ), muito baixo (entre $50 \mathrm{e}$ $200 \mathrm{~L} / \mathrm{ha}$ ) e ultrabaixo (menos de $50 \mathrm{~L} / \mathrm{ha}$ ). Nas regiões produtoras de frutas, na Europa, estão sendo utilizadas aplicações com baixo e muito baixo volume, enquanto no sul do Brasil variam de médio a alto.

Cross \& Berrie (1990) citam que o método de aplicação com ultrabaixo volume produz grande quantidade de gotas pequenas com pouca variação de tamanho, fato que não ocorre nas aplicações com os volumes baixo e médio. Por esse motivo, o ultrabaixo volume favorece a melhor deposição do produto sobre a folha, conseguindo-se, desta forma, melhor controle das pragas e das doenças. Herrera-Aguirre \& Unrath (1980), ao contrário, afirmam que o raleio de frutos ocorre somente quando os ramos são bem molhados, o que enfatiza a necessidade da aplicação do tratamento até o ponto próximo do escorrimento.

Este trabalho teve o objetivo de comparar a eficiência de diferentes volumes de calda de raleantes químicos, aplicados em plantas de macieira, cultivar Gala.

\section{MATERIAL E MÉTODOS}

O experimento foi realizado em pomar comercial de macieira, cultivar Gala/MM106, instalado em 1987, no espaçamento de 5,0 m x 1,5 m, no município de Fraiburgo, Santa Catarina, e conduzido durante três anos (1993 a 1995).

Os raleantes químicos utilizados foram o ácido naftalenoacético (ANA) e o carbaryl, aplicados, seqüencialmente, aos 5 e aos 15 dias após a plena floração (DAPF), respectivamente. As dosagens dos raleantes químicos por área, em termos de ingredientes ativos, foram as mesmas em todos os tratamentos. No caso do ANA, usaram-se $75 \mathrm{~g} / \mathrm{ha}$ do produto comercial com $20 \%$ de ingrediente ativo (i.a.), nos três anos de experimentação. Quanto ao carbaryl, em 1993 usou-se uma formulação pó-molhável, com $85 \%$ de i.a., aplicando-se $1.180 \mathrm{~g} / \mathrm{ha}$ do produto comercial. Nos dois anos seguintes, passou-se à formulação de suspensão concentrada, com $48 \%$ de i.a., aplicando-se $2.100 \mathrm{~mL} / \mathrm{ha}$ do produto comercial. Essas dosagens foram calculadas com base na recomendação de raleio para a culti- var Gala (7,5 ppm para o ANA e 500 ppm para o carbaryl, ajustadas para $1.900 \mathrm{~L} / \mathrm{ha}$ ). A mudança na formulação do carbaryl foi motivada pela dificuldade da sua aplicação no tratamento $16 \mathrm{~L} / \mathrm{ha}$, no qual, praticamente, toda a calda se transformava em espuma, causando falha na aplicação. A mudança para a formulação suspensão concentrada melhorou a aplicação, embora não tenha solucionado totalmente o problema.

Em todos os tratamentos com raleantes químicos, com exceção da formulação líquida de carbaryl, foi adicionado óleo mineral emulsionável, na diluição de $250 \mathrm{~mL} / 100 \mathrm{~L}$ d'água.

O delineamento estatístico foi inteiramente casualizado, com nove tratamentos e quatro repetições de duas plantas por parcela. A aplicação foi feita em três fileiras paralelas de plantas, avaliando-se somente as da fila central.

Os tratamentos foram os seguintes: 1) $1.900 \mathrm{~L} / \mathrm{ha}$, aplicado com pulverizador manual estacionário, utilizando-se pistola com bico tipo cone vazio; 2) testemunha (sem tratamento); 3) raleio manual seletivo feito aos $30 \mathrm{DAPF}$, deixando-se duas frutas/cacho floral quando o ramo novo proveniente da inflorescência fosse igual ou superior a $10 \mathrm{~cm}$ e apenas uma fruta/cacho quando inferior a $10 \mathrm{~cm}$ ou na ausência desse ramo, mas com folhas de tamanho normal junto à inflorescência. Quando não havia crescimento novo e as folhas eram pequenas, todas as frutas foram retiradas; 4) $300 \mathrm{~L} / \mathrm{ha}$, usando-se um turboatomizador marca Agrotécnica com bicos tipo Albuz 212-4; 5) 430 L/ha, usando-se um turboatomizador marca Jacto com bicos tipo JA-2; 6) $950 \mathrm{~L} / \mathrm{ha}$, usando-se um turboatomizador marca Jacto com bicos tipo J5-2; 7) $1.300 \mathrm{~L} / \mathrm{ha}$, usando-se um turboatomizador marca Agrotécnica com bicos tipo 3,9-4; 8) 1.900 L/ha, usandose um turboatomizador marca Jacto com bicos J5-3; 9) $16 \mathrm{~L} / \mathrm{ha}$, usando-se um turboatomizador marca Agrotécnica com bicos rotativos.

Os tratamentos com turboatomizador foram aplicados com trator $75 \mathrm{HP}$, na velocidade de $3,9 \mathrm{~km} / \mathrm{h}$ no tratamento com $16 \mathrm{~L} / \mathrm{ha}$, e com a velocidade de $5,6 \mathrm{~km} / \mathrm{h}$ nos demais tratamentos. A pressão de trabalho foi de $120 \mathrm{lb} / \mathrm{pol}^{2}$ no tratamento 1 , de $200 \mathrm{lb} / \mathrm{pol}^{2}$ nos tratamentos com turboatomizador marca Jacto, e de $90 \mathrm{lb} / \mathrm{pol}^{2}$ nos tratamentos realizados com turboatomizador marca Agrotécnica.

A quantidade de litros por hectare depende da vazão dos bicos, da pressão de trabalho da bomba, e da velocidade de trabalho do turboatomizador. Daí a necessidade de se trabalhar com bico, pressão e velocidade variáveis. Os dois tipos de turboatomizadores são bastante usados na região produtora de maçãs do sul do Brasil.

As avaliações foram realizadas coletando-se todas as frutas por parcela, separando-as em sete classes, de acordo com o diâmetro $(\leq 56 \mathrm{~mm}, 57-61 \mathrm{~mm}, 62-65 \mathrm{~mm}$, 66-69 mm, 70-76 mm, 77-84 mm e $\geq 85 \mathrm{~mm}$ ). As frutas foram contadas e pesadas por classe. $\mathrm{Na}$ avaliação da inci- 
dência de "russeting", utilizou-se uma amostra de 100 frutas/parcela, ao acaso, atribuindo-se graus numa escala de 1-5, representando: grau 1, a ausência de "russeting" nas frutas, com exceção daquele restrito à cavidade peduncular; 2, as frutas com até $10 \%$ da superfície com "russeting"; 3 , com $10 \%$ a $30 \%$; 4 , com $30 \%$ a $50 \%$; 5 , com mais de $50 \%$. Na análise do porcentual de "russeting", foi utilizada a fórmula de Townsend \& Heuberger (1943). Os dados foram submetidos a análise de variância, e as médias foram comparadas pelo teste de Duncan, a $5 \%$ de probabilidade.

\section{RESULTADOS E DISCUSSÃO}

Em virtude da ocorrência de interação entre os fatores ano e tratamento não foi feita análise conjun- ta dos três ciclos em que o experimento foi conduzido.

$\mathrm{O}$ peso dos frutos (Tabela 1) e o porcentual de frutos com diâmetro igual ou acima de $66 \mathrm{~mm}$ (Tabela 2) são os dois parâmetros mais importantes para medir o efeito dos tratamentos de raleio. Frutos maiores e de melhor coloração em virtude do raleio obtêm melhor preço no mercado. Em todos os tratamentos, com exceção de $430 \mathrm{~L} / \mathrm{ha}$, o peso dos frutos foi maior do que o da testemunha. No entanto, o aumento do peso dos frutos que ficaram na planta foi insuficiente para compensar os frutos retirados. Embora os tratamentos apresentassem valores de produtividade média acima de $40.000 \mathrm{~kg} / \mathrm{ha}$ - considerada boa produtividade para a cultivar Gala, nas condições do sul brasileiro -, foram inferiores aos da testemunha (Tabela 1).

TABELA 1. Peso dos frutos no período de 1993 a 1995 e produção média desses três anos, em plantas de macieira, cultivar Gala, tratados com diferentes volumes de calda de raleantes químicos ${ }^{1}$.

\begin{tabular}{|c|c|c|c|c|c|}
\hline \multirow[t]{2}{*}{ Tratamento } & \multicolumn{4}{|c|}{ Peso dos frutos $(\mathrm{g})$} & \multirow{2}{*}{$\begin{array}{c}\text { Produção Média } \\
(\mathrm{kg} / \mathrm{ha})\end{array}$} \\
\hline & 1993 & 1994 & 1995 & Média & \\
\hline Testemunha & $113 d$ & $95 \mathrm{~d}$ & $80 \mathrm{~d}$ & 96,0 & 68.724 \\
\hline Raleio manual & $122 \mathrm{~cd}$ & $110 \mathrm{bc}$ & $100 \mathrm{ab}$ & 110,7 & 47.387 \\
\hline $16 \mathrm{~L} / \mathrm{ha}$ & $131 \mathrm{abc}$ & $121 \mathrm{a}$ & $91 b c$ & 114,3 & 45.037 \\
\hline $300 \mathrm{~L} / \mathrm{ha}$ & $134 \mathrm{ab}$ & $109 \mathrm{bc}$ & $94 b c$ & 112,3 & 67.936 \\
\hline $430 \mathrm{~L} / \mathrm{ha}$ & $122 \mathrm{~cd}$ & $100 \mathrm{~cd}$ & $88 \mathrm{~cd}$ & 103,3 & 48.313 \\
\hline $950 \mathrm{~L} / \mathrm{ha}$ & $128 \mathrm{abc}$ & $107 \mathrm{bc}$ & $92 b c$ & 109,0 & 59.505 \\
\hline $1300 \mathrm{~L} / \mathrm{ha}$ & $138 \mathrm{a}$ & $115 \mathrm{ab}$ & $104 a$ & 119,0 & 63.037 \\
\hline $1.900 \mathrm{~L} / \mathrm{ha}$ com pulverizador & $125 \mathrm{bc}$ & $106 \mathrm{bc}$ & $94 b c$ & 108,4 & 64.316 \\
\hline $1.900 \mathrm{~L} /$ ha com turboatomizador & $133 \mathrm{ab}$ & $109 \mathrm{bc}$ & $95 \mathrm{abc}$ & 112,3 & 64.129 \\
\hline $\mathrm{CV}(\%)$ & 4,8 & 6,4 & 6,1 & - & - \\
\hline $\mathrm{F}$ (tratamento) & $0,0002 * *$ & $0,0011 *$ & $0,0004 * *$ & - & - \\
\hline
\end{tabular}

${ }^{1}$ Médias seguidas pela mesma letra, dentro de cada coluna, não diferem entre si pelo teste de Duncan a 5\% de probabilidade.

* e ** Significativo a $5 \%$ e $1 \%$ de probabilidade, respectivamente.

TABELA 2. Porcentagem da massa de frutos com diâmetro igual ou superior a $66 \mathrm{~mm}$, no período de 1993 a 1995 , em plantas de macieira, cultivar Gala, tratadas com diferentes volumes de calda de raleantes químicos ${ }^{1}$.

\begin{tabular}{|c|c|c|c|c|}
\hline \multirow[t]{2}{*}{ Tratamento } & \multicolumn{4}{|c|}{ Porcentagem da massa de frutos ${ }^{2}$} \\
\hline & 1993 & 1994 & 1995 & Média \\
\hline Testemunha & $49,5 \mathrm{e}$ & $24,1 d$ & $5,6 \mathrm{~d}$ & 26,4 \\
\hline Raleio manual & $67,3 \mathrm{~cd}$ & $48,4 \mathrm{abc}$ & $25,0 \mathrm{~b}$ & 46,9 \\
\hline $16 \mathrm{~L} / \mathrm{ha}$ & $77,3 \mathrm{abc}$ & $64,9 \mathrm{a}$ & $17,3 \mathrm{bc}$ & 53,2 \\
\hline $300 \mathrm{~L} / \mathrm{ha}$ & $79,7 \mathrm{ab}$ & $47,6 a b c$ & $17,1 \mathrm{bc}$ & 48,1 \\
\hline $430 \mathrm{~L} / \mathrm{ha}$ & $64,5 \mathrm{~d}$ & $35,6 \mathrm{~cd}$ & $12,4 \mathrm{~cd}$ & 37,5 \\
\hline $950 \mathrm{~L} / \mathrm{ha}$ & $73,9 \mathrm{abcd}$ & $47,8 \mathrm{abc}$ & $18,5 \mathrm{bc}$ & 46,7 \\
\hline $1.300 \mathrm{~L} / \mathrm{ha}$ & $82,3 \mathrm{a}$ & $55,6 \mathrm{ab}$ & $38,8 \mathrm{a}$ & 58,9 \\
\hline $1.900 \mathrm{~L} / \mathrm{ha}$ com pulverizador manual & $69,7 \mathrm{bcd}$ & $46,6 b c$ & $17,8 \mathrm{bc}$ & 44,7 \\
\hline 1. $900 \mathrm{~L} / \mathrm{ha}$ com turboatomizador & $80,0 \mathrm{ab}$ & $48,1 \mathrm{abc}$ & $22,3 \mathrm{bc}$ & 50,1 \\
\hline $\mathrm{CV}(\%)$ & 9,4 & 23,0 & 37,8 & \\
\hline $\mathrm{F}$ (tratamento) & $0,0001 * *$ & $0,0017 *$ & $0,0002 * *$ & \\
\hline
\end{tabular}

${ }^{1}$ Médias seguidas pela mesma letra, dentro de cada coluna, não diferem entre si pelo teste de Duncan a 5\% de probabilidade.

${ }^{2}$ A porcentagem de massa foi determinada em relação ao peso de frutos.

* e ** Significativo a $5 \%$ e $1 \%$ de probabilidade, respectivamente. 
Os tratamentos com volumes de 16, 300, 950, 1.300 e $1.900 \mathrm{~L} / \mathrm{ha}$ apresentaram porcentual de frutas $\geq 66 \mathrm{~mm}$ de diâmetro (média dos três anos), aproximadamente igual ao dobro do da testemunha (Tabela 2). A aplicação seqüencial de ANA e de carbaryl foi eficiente no raleio de maçãs, fato já comprovado anteriormente por Ebert et al. (1988) e Camilo et al. (1991, 1992), mas não houve diferenças entre os volumes de calda testados. Palladini \& Reis Filho (1996), usando cinco dos nove volumes testados neste experimento $(16,300,430,950$ e $1.300 \mathrm{~L} / \mathrm{ha}) \mathrm{e}$ os mesmos equipamentos, para o controle do ácarovermelho-europeu (Panonychus ulmi), obtiveram o mesmo nível de controle, independentemente do volume utilizado.

A efetividade do tratamento com ultrabaixo volume (16 L/ha) pode ser atribuída ao fato de que o bico rotativo produz gotas de tamanho uniforme. Cross \& Berrie (1990) citaram que os bicos rotativos, produzindo gotas entre 60 e 120 micra, proporcionam melhor distribuição do raleante químico e melhor cobertura da planta, ao mesmo tempo que reduzem ou eliminam a perda por escorrimento. Oakford et al. (1995), testando volumes de calda baixos, médios e altos, obtiveram melhores resultados no raleio de frutas com os volumes mais baixos, mesmo quando a quantidade de raleante por hectare foi reduzida em $25 \%$ e $50 \%$ em relação à normal. Aqueles autores atribuem os resultados à melhor distribuição dos produtos nas plantas, graças ao tamanho das gotas geradas pelos equipamentos usados para baixos volumes. Os dados apresentados neste trabalho também estão de acordo com os resultados obtidos por Black et al. (1995), que conseguiram resultados efetivos no raleio de maçãs com qualquer um dos volumes testados $(250,1.000$ e $2.000 \mathrm{~L} / \mathrm{ha})$ quando a quantidade em i.a./ha do raleante foi fixada.

Uma vez que os diferentes volumes de calda foram igualmente eficientes no raleio de frutos, acredita-se que o mais importante seja a quantidade de raleante que atinge as folhas, as flores e os frutos em desenvolvimento. Se volumes mais baixos podem ser tão ou mais eficientes quanto os volumes altos, isso pode representar vantagens do ponto de vista prático. Uma das vantagens é a redução do tempo de aplicação dos tratamentos.

Palladini \& Kreuz (1995) determinaram que a redução do volume de calda de $1.100 \mathrm{~L} /$ ha para $550 \mathrm{~L} / \mathrm{ha}$ diminuiu em 35,19\% o tempo gasto na aplicação do tratamento.

Durante a época do raleio, é freqüente ocorrerem períodos chuvosos. E mesmo após a chuva, há necessidade de se esperar algum tempo para que o solo permita a entrada do trator com o turboatomizador no pomar, o que torna a aplicação dos tratamentos, nos grandes pomares, um sério problema. Com a perspectiva de se usarem volumes mais baixos de calda, pode-se diminuir o número de reabastecimentos do turboatomizador, e com isso, reduzir o tempo de aplicação, racionalizando melhor o uso das máquinas e equipamentos. $\mathrm{O}$ uso de volumes mais baixos de calda pode, também, contribuir para a redução da poluição ambiental, e diminuir ou eliminar totalmente o escorrimento do produto para o solo.

Não houve efeito do volume de calda sobre a incidência de "russeting" (Tabela 3), confirmando-

TABELA 3. Porcentagem de 'russeting' sobre o fruto, no período de 1993 a 1995 , em plantas de macieira, cultivar Gala, tratadas com diferentes volumes de calda de raleantes químicos.

\begin{tabular}{|c|c|c|c|c|}
\hline \multirow[t]{2}{*}{ Tratamento } & \multicolumn{4}{|c|}{ Porcentagem de 'russeting' nos frutos } \\
\hline & 1993 & 1994 & 1995 & Média \\
\hline Testemunha & 24,1 & 23,0 & 13,0 & 20,0 \\
\hline Raleio manual & 26,5 & 27,3 & 15,2 & 23,0 \\
\hline $16 \mathrm{~L} / \mathrm{ha}$ & 24,2 & 26,1 & 20,8 & 23,7 \\
\hline $300 \mathrm{~L} / \mathrm{ha}$ & 23,6 & 23,5 & 15,8 & 21,0 \\
\hline $430 \mathrm{~L} / \mathrm{ha}$ & 22,9 & 25,0 & 18,3 & 22,1 \\
\hline $950 \mathrm{~L} / \mathrm{ha}$ & 23,3 & 27,2 & 15,3 & 21,9 \\
\hline $1.300 \mathrm{~L} / \mathrm{ha}$ & 29,1 & 25,7 & 19,7 & 25,7 \\
\hline $1.900 \mathrm{~L} / \mathrm{ha}$ com pulverizador manual & 29,3 & 26,3 & 14,3 & 23,3 \\
\hline $1.900 \mathrm{~L} / \mathrm{ha}$ com turboatomizador & 26,0 & 25,9 & 15,9 & 22,6 \\
\hline $\mathrm{CV}(\%)$ & 18,4 & 8,2 & 23,3 & - \\
\hline $\mathrm{F}$ tratamento & $0,41^{\mathrm{ns}}$ & $0,09^{\mathrm{ns}}$ & $0,4^{\mathrm{ns}}$ & - \\
\hline
\end{tabular}

ns Não-significativo. 
se, assim, resultados anteriormente obtidos por Camilo et al. (1991, 1992).

Pelos resultados obtidos por Oakford et al. (1995), também seria possível a redução das dosagens dos raleantes químicos, quando estes são aplicados em volumes menores de calda; mas esse é um item a ser considerado em futuras pesquisas nas condições do sul do Brasil.

\section{CONCLUSÕES}

1. Os diferentes volumes de calda, ultrabaixo, baixo, médio e alto contendo as mesmas quantidades de ingrediente ativo dos raleantes apresentam eficiência similar no raleio de frutas da macieira 'Gala'.

2. O volume de calda não é o fator mais importante para a eficiência dos raleantes químicos ácido naftalenoacético e carbaryl.

\section{REFERÊNCIAS}

BLACK, B.L.; BUKOVAC, M.J.; HULL JUNIOR, J. Effect of spray volume and time of NAA application on fruit size and cropping of Redchief Delicious apple. Scientia Horticulturae, Amsterdam, v.64, n.4, p.253-264, 1995.

CAMILO, A.P.; DENARDI, F.; ZAFFARI, G.R.; KREUZ, C.L. Raleio de frutos na cultivar Golden Delicious. Revista Brasileira de Fruticultura, Cruz das Almas, v.14, n.1, p.89-96, 1992.

CAMILO, A.P.; MONDARDO, M.; LEITE, G.B. Raleio de frutos de macieira, cultivar Gala. Revista Brasileira de Fruticultura, Cruz das Almas, v.13, n.1, p.145-152, 1991.

CROSS, J.V.; BERRIE, A.M. Efficacy of reduced volume and reduced dose rate spray programmes in apple orchards. Crop Protection, Oxford, v.9, n.3, p.207$217,1990$.

EBERT, A.; KREUZ, C.L.; ZAFFARI, G.R.; PETRI, J.L. Raleio de frutos em macieira no alto vale do Rio do Peixe em Santa Catarina. Florianópolis : EMPASC, 1988. 65p.

EDGERTON, L.J. Chemical thinning of flowers and fruit. In: KOZLOWSKI, T.T. (Ed.). Shedding of plant parts. New York : Academic, 1973. p.435-474.
ESPADA CARBÓ, J.L. El aclareo químico: aplicación a flores y jóvenes frutos de manzano. Hortofruticultura, Madrid, v.5, n.4, p.53-55, 1994.

FAUST, M. Physiology of temperate zone fruit trees. New York : J. Wiley, 1989. 338p.

GIULIVO, C.; RAMINA, A.; COSTA, G. Metabolism and translocation of $1,2^{-14} \mathrm{C}$ 2-chloroethyl-phosphonic acid in Prunus persica (L.) Batsch. Scientia Horticulturae, Amsterdam, v.15, n.1, p.33-43, 1981.

HERRERA-AGUIRRE, E.; UNRATH, C.R. Chemical thinning response of 'Delicious' apples to volume of applied water. HortScience, Alexandria, v.15, n.1, p.43-44, 1980.

MATTHEWS, G.A. Pesticide application methods. London : Longman, 1982. 336p.

NIR, G.; LAVEE, S. Persistence, uptake and translocation of ${ }^{14} \mathrm{C}$ ethephon (2-chloroethylphosphonic acid) in Perlette and Cardinal grapevines. Australian Journal of Plant Physiology, Collingwood, v.8, n.1, p.5763, 1981.

OAKFORD, M.J.; BOUND, S.A.; JONES, K.M.; O'RIELLY, L.O. Use of airshear technology to reduce chemical spray rates for thinning of apples. Australian Journal of Experimental Agriculture, Collingwood, v.35, n.6, p.789-794, 1995.

PALLADINI, L.A.; KREUZ, C.L. Redução dos custos de produção da macieira com médio volume de calda nos tratamentos fitossanitários. Agropecuária Catarinense, Florianópolis, v. 8, n.1, p.48-49, 1995.

PALLADINI, L.A.; REIS FILHO, W. Eficiência de diferentes volumes de calda no controle do ácaro Panonychus ulmi (Koch) da macieira. Anais da Sociedade Entomológica do Brasil, Viçosa, v.25, n.1, p.161-164, 1996.

TOWNSEND, G.R.; HEUBERGER, J.W. Methods for estimating losses caused by diseases in fungicides experiments. Plant Disease Reporter, St. Paul, v.27, n.17, p.340-343, 1943.

WERTHEIM, S.J. Chemical thinning of Golden Delicious apple with NAAm and/or carbaryl in combination with a spreader and the anti-russeting agent $\mathrm{GA}_{7+4}$. Acta Horticulturae, Leuven, n.179, p.659-666, 1986. 